\title{
COMMISSION 7: CELESTIAL MECHANICS (MÉCANIQUE CÉLESTE)
}

President: S. Ferraz-Mello

Vice-President: Cl. Froeschlé

Organizing Committee: R. Dvorak, K.V. Kholshevnikov, H. Kinoshita, J.H. Lieske, A. Milani, J.C. Muzzio, S.J. Peale, A.E. Roy, M. Soffel, A.G. Sokolsky, Y.-S. Sun

Since the last report, Commission 7 has supported IAU Symposium No. 172 "Dynamics, Ephemerides and Astrometry of the Solar System" (Paris, 1995) and co-supported IAU Colloquium No. 165 "Dynamics and Astrometry of Natural and Artificial Celestial Bodies" (Poznań, Poland, July 1996). During the 22 General Assembly (The Hague, 1994), the Commission co-supported the joint discussions "Time scales: state of the art" and "Nutations" as well as the working group meeting "Near-Earth Objects Detection".

Other international conferences with important participation of Commission 7 members were: "Modern Problems of Theoretical Astronomy" (St.Petersburg, Russia, June 1994); "Small Bodies in the Solar System and their Interaction with the Planets" (Mariehamm, Finland, August 1994); "The NATO Advanced Study Institute "Hamiltonian Systems with 3 or more Degrees of Freedom (3DHAM95)" (S'agaró, Spain, June 1995); "Chaos in Gravitational N-body Systems" (La Plata, Argentina, July 1995); $4^{\text {th }}$ Alexander von Humboldt Colloquium on Celestial Mechanics: "The Dynamical Behaviour of our Planetary System" (Ramsau, Austria, April 1996); "Kuiper Belt Workshop" (Toronto, Canada, June 1996); "Asteroids, Comets, Meteors" (Versailles, France, July 1996); "Visual Double Stars: Formation, Dynamics and Evolutionary Tracks" (Santiago de Compostela, Spain, July 1996); "4 ${ }^{\text {th }}$ Workshop on Positional Astronomy and Celestial Mechanics" (Penyiscola, Spain, October 1996).

Several regional or national meetings centered on Celestial Mechanics and Dynamical Astronomy were also held during the past three years: the $25^{\text {th }}, 26^{\text {th }}$ and $27^{\text {th }}$ annual meetings of the Division on Dynamical Astronomy of the American Astronomical Society (Kingsville, March 1994; Yosemite, April 1995; Washington, April 1996); the $26^{\text {th }}, 27^{\text {th }}$ and $28^{\text {th }}$ Japanese Symposiums on Celestial Mechanics (Tokyo, January 1994, January 1995 and January 1996); the $7^{\text {th }}$ and $8^{\text {th }}$ Brazilian Colloquiums on Orbital Dynamics (Santos, November 1994 and November 1996); the Winter Schools "Chaos and Diffusion in Hamiltonian Systems" (Chamonix, February 1994) and "Discrete Dynamical Systems - Mappings" (Aussois, February 1996); the workshop "Perturbation Theory and Chaos in Nonlinear Dynamics with Emphasis to Celestial Mechanics" (Funchal, Madeira, May 1994).

Several books dealing with Celestial Mechanics were published since the last report:

- Benest, D. \& Froeschlé, Cl.(eds.): 1995, "Chaos and Diffusion in Hamiltonian Systems", Éditions Frontières, Gif-sur-Yvette.

- Boccaletti, D. \& Pucacco, G.: 1996, "Theory of Orbits. Vol 1. Integrable Systems and Non-perturbative Methods", Springer-Verlag.

- Brumberg, V.A.: 1995, "Analytical Techniques of Celestial Mechanics", Springer-Verlag.

- Celletti, A. et al (eds.): 1993, "Primo Convegno Nazionale di Meccanica Celeste", Dip. Matemat. Univ. L'Aquila, L'Aquila.

- Contopoulos, G., Spyrou, N.K. \& Vlahos, L. (eds.): 1994, "Galactic Dynamics and N-body Simulations", Springer-Verlag.

- Ferraz-Mello, S., Morando, B. \& Arlot, J.-E. (eds.): 1996, "Dynamics, Ephemerides and Astrometry of the Solar System", Proceedings of IAU Symposium No. 172, Kluwer, Dordrecht.

- Gurzadyan, V.G. \& Pfenniger, D. (eds.): 1994, "Ergodic Concepts in Stellar Dynamics", SpringerVerlag.

- Hagel, J., Cunha, M. \& Dvorak, R. (eds.): 1995, "Perturbation Theory and Chaos in Nonlinear Dynamics with emphasis to Celestial Mechanics", Universidade da Madeira, Funchal \& Institut für Astronomie, Vienna.

- Hut, P. (ed.): 1996, "Dynamical Evolution of Star Clusters - Confrontation of Theory and Observations", Kluwer, Dordrecht.

- Kandrup, H.E., Gottesman, S.T. \& Ipser, J.R. (eds.): 1995, "Three-Dimensional Systems", Ann. N.Y. Acad. Sc. 751, New York. 
- Kinoshita, H. \& Nakai, H. (eds.): 1994, "Proceedings of the $26^{\text {th }}$ Symposium on Celestial Mechanics", Tokyo.

- Kinoshita, H. \& Nakai, H. (eds.): 1995, "Proceedings of the $27^{\text {th }}$ Symposium on Celestial Mechanics", Tokyo.

- Kinoshita, H. \& Nakai, H. (eds.): 1996, "Proceedings of the $28^{\text {th }}$ Symposium on Celestial Mechanics", Tokyo.

- Kurzyńska, K., Barlier, F., Seidelmann, P.K. \& Wytrzyszczak, I. (eds.): 1994, "Dynamics and Astrometry of Natural and Artificial Celestial Bodies", Astr. Observ., Poznań.

- Lacomba, E. \& Llibre, J. (eds.): 1996, "New trends in Hamiltonian Systems and Celestial Mechanics", World Scientific, Singapore.

- Lopez Garcia, A. et al (eds.): 1993, "Proceedings of the $2^{\text {nd }}$ International Workshop on Positional Astronomy and Celestial Mechanics", Obs. Astron., Valencia.

- Muzzio, J.C., Ferraz-Mello, S. \& Henrard, J. (eds.): 1996, "Chaos in Gravitational N-body Systems", Kluwer, Dordrecht.

- Rickman, H. \& Valtonen, M.J. (eds.): 1996, "Worlds in Interaction: Small Bodies and Planets of the Solar System", Kluwer, Dordrecht.

- Roy, A.E. \& Steves, B.A. (eds.): 1995, "From Newton to Chaos: Modern Techniques of Understanding and Coping with Chaos in N-Body Dynamical Systems", NATO Adv. Stud. Inst. Ser. B Phys. 336, Plenum Press, New York.

- Seimenis, J. (ed.): 1993, "Hamiltonian Mechanics: Integrability and chaotic behaviour", NATO Adv. Stud. Inst. Ser. B Phys. 331, Plenum Press, New York.

As for the previous reports, the Organizing Committee of Commission 7 decided that some of the chapters of Celestial Mechanics in which significant progress was observed in the past three years should be selected and specialists asked to report on it. The selection aimed at showing the multiple directions of development of modern Celestial Mechanics with emphasis in some domains which are new frontiers of interest in this discipline. It also included one report, extending over a larger timespan, on the impact of modern Mathematics in Celestial Mechanics, an important subject that was never been reviewed in these reports. Later on, a report on the motions of the Moon was included, in order to account for the remarkable advances recorded in our knowledge of these motions, in special the Moon's librations. Some other subjects considered in the previous triennial reports as, for instance, Solar System chaos and the dynamics of asteroidal motion, continue to record significant progress but were not included in the report this time only because of the limited space available for it.

Informations, abstracts and copies of many papers in Celestial Mechanics and Dynamical Astronomy are systematically released at the web sites http://e-math.ams.org (MathSciNet database of the American Mathematical Society) and http://adsabs .harvard .edu (Nasa Astrophysics Data System). Informations and tables of contents of the journal Celestial Mechanics and Dynamical Astronomy (CMDA) are released in the web site http://ww. wkap.nl. An index of that journal is available through anonymous ftp at arzach.info.fundp.ac.be (ID 138.48.4.5), directory pub/cel_mech.

\section{A. Dynamics of the Kuiper belt}

(A. Morbidelli)

Since the pioneer works by Torbett and Smoluchowski (Nature 345, 49), Holman and Wisdom (AJ 105, 1987) and Levison and Duncan (ApJ 406, L35), the interest in the dynamics of the Kuiper belt has been constantly increasing. On the one hand, the above quoted papers showed that the Kuiper belt has a rich and puzzling dynamical structure, characterized by a sequence of stable and unstable regions within a few Astronomical Units; on the other hand, thanks to the discovery of new objects (32 up to the present time), we have now a first hint on the real distribution of Kuiper belt members, which challenges our understanding. The dynamics in the Kuiper belt has been a subject of large debate at the latest KB workshop and ACM Colloquium.

\section{THE PRESENT DYNAMICAL STRUCTURE OF THE KUIPER BELT}

The dynamical properties of the Kuiper belt (KB) have been investigated analytically by Morbidelli, Thomas and Moons (Icarus 118, 322). They computed the location and the width of the main mean- 
motion resonances with Neptune and Uranus and pointed out the existence of a sequence of resonances of first order with Neptune $(5 / 6,4 / 5,3 / 4,2 / 3)$ which seem to be correlated to the regions that Holman and Wisdom (AJ 105, 1987) and Levison and Duncan (ApJ 406, L35) found to be stable over a 1Gyr timescale in their numerical integrations. Their analytic computations are based on the averaged planar circular three body problem. At the same time, Malhotra (AJ 111, 504) determined the size of the regular regions in mean-motion resonances, computing Poincaré sections of the non-averaged planar circular three body problem. As a consequence, the widths of the resonant regions computed by Malhotra are somewhat smaller than the ones computed by Morbidelli et al, because of the presence of chaotic layers, in the non-averaged problem. As a matter of fact, taking into account the eccentricity of Neptune's orbit and its secular oscillations, the regular regions should be even smaller than what computed by Malhotra, although no quantitative computation has been performed up to now.

Morbidelli et al gave a simple explanation of the fact that mean-motion resonances of order one should be stable over at least long times: due to the form of the leading term in the perturbation function, the longitude of perihelion of resonant orbits precesses with a fast frequency (roughly proportional to $1 / e, e$ being the eccentricity of the orbit). Therefore, there cannot be secular resonances between the frequency of the longitude of perihelion of the resonant body and the frequencies of the perihelia of the planets, so that the eccentricity can have only fast and small oscillations, conversely to what happens in most of the Kirkwood gaps in the asteroid belt. In first-order mean-motion resonances in the Kuiper belt, secular resonances of perihelia can exist only at very large amplitude of libration or at large eccentricity. Conversely, secular resonances involving the nodes can exist also at small eccentricity, so that the inclination can show slow and large oscillations.

Orbits in any mean-motion resonance with Neptune are phase protected from encountering the planet even if their perihelion is well inside Neptune's orbit, as in the case of Pluto. Conversely, orbits outside mean-motion resonances are not phase protected, so that they are unstable due to Neptune close encounters as soon as the perihelion distance $q$ is close to $30 \mathrm{AU}$. Taking into account the secular oscillation of the eccentricity, this implies that non-resonant orbits with initial eccentricity above some threshold, dependent on the semi-major axis, become unstable very quickly with respect to the age of the Solar System. This explains why the stability times drop between mean-motion resonances, a feature well visible in the figures of Holman and Wisdom (AJ 105, 1987) and Levison and Duncan (ApJ 406, L35). The instability is particularly strong between 35-36 AU and between $40-42 \mathrm{AU}$, due to the presence of the secular resonances $\nu_{7}$ and $\nu_{8}$ (in the first case), and $\nu_{8}$ and $\nu_{18}$ (in the second case). The location of these secular resonances has been first determined by. Knežević et al (Icarus 93, 316). Morbidelli et al have studied with simple models their effects and showed that their overlapping gives origin to large scale chaos where the eccentricity, even if initially equal to zero, is forced to increase to Neptune encountering values.

On the side of numerical explorations, the early work by Levison and Duncan (ApJ 406, L35) has been improved by Duncan, Levison and Budd (AJ 110, 3073). The fate of thousands of orbits with initial conditions chosen over a regular grid in the $a-e-i$ space has been investigated over $4 \mathrm{Gyr}$. The dynamical structure of the KB is pointed out in very readable color pictures. The main results of this paper can be summarized as follows.

- The limit of stability of orbits outside mean-motion resonances in the $a-e$ plane is well determined and follows approximately the curve $q=35 \mathrm{AU}$ (although dropping to $e=0$ in the secular resonance region at 35-36 and 40-42 AU). This implies that orbits with semi-major axis between 36 and 40 $\mathrm{AU}$ and initial eccentricity smaller than 0.05 are stable over the age of the Solar System. Puzzling enough, no objects have been discovered in this region (see the next section for discussion).

- Stable regions over the age of the Solar System are found associated to all mean-motion resonances of first order, even at large eccentricity. However, these resonant stable regions seem to disappear at large inclination, a feature still not well understood.

- Regions characterized by very slow instability are found to be of non negligible size. Test particles in these regions become Neptune encounters only after billions of years of integration. The dynamical nature of these orbits is not understood, however their great importance for the origin of comets is pointed out. Indeed, believing that the Kuiper belt is the reservoir of short-periodic (SP) comets and taking into account that the latter have a short dynamical and physical lifetime, one can conclude that the SP comets that we observe now must have left the KB just a few millions of years ago, 
namely some billions of years after their formation. As a consequence, the presently observed SP comets should come only from the regions of slow instability in the KB.

- Having a numerical estimate of the relative volume of such slowly unstable regions, Duncan, Levison and Budd (op cit) determined that there should be about $10^{10}$ comet-sized bodies in the Kuiper Belt in order to explain the presently observed number of Jupiter family comets. This is the first estimate of the population of small bodies in the KB which, together with the estimates of the population of big bodies computed from the discovery rate in observational surveys, allows to derive a sort of preliminary size-distribution law of bodies in the Kuiper Belt.

Understanding the dynamical nature of slowly unstable orbits is the main challenge for Celestial Mechanics in the next years. A preliminary result has been presented by Morbidelli Thomas and Moons (Icarus subm.) concerning slow instabilities in the $2 / 3$ resonance with Neptune. The resonance is found to be characterized by a smooth transition from regular orbits (invariant KAM tori), at small amplitude of libration, and strong chaos forced by the overlapping of the $\nu_{18}$ and Kozai resonances, at large amplitude of libration. As a consequence, orbits with all possible escape times can be found.

\section{THE DISTRIBUTION OF KB OBJECTS AND THE EARLY HISTORY OF THE BELT}

Although some of the 32 discovered KB objects have been observed during more than one opposition, the orbit of none of them has still been determined with an accuracy comparable to that of the orbits of numbered asteroids. As a consequence, a detailed investigation of the dynamical behaviour of real objects cannot be done yet, because the uncertainty on the orbital elements is still too important. It should not be forgotten that, in most cases, among all possible orbital solutions compatible with the observations, the selected one is usually that which turns out to be the most stable in long-term numerical integrations.

Nevertheless, some considerations on the distribution of bodies in the KB can be derived:

1. All objects with $a$ smaller than $40 \mathrm{AU}$ are in mean-motion resonances with Neptune (the vast majority are in the $2 / 3$ resonance) and have a large eccentricity $(e>0.1$; in some cases $e>0.3$ ).

2. No objects have been discovered at small eccentricity in non-resonant orbits between 36 and $40 \mathrm{AU}$, despite of the fact that these orbits are stable over the age of the Solar System.

3. No objects have been discovered in the $1 / 2$ resonance with Neptune.

4. The objects with $a>42 \mathrm{AU}$ have small eccentricity (most have $e<0.1$ ) and do not seem to be associated specifically to mean-motion resonances.

A nice model to explain point (1) above has been proposed by Malhotra (AJ 110, 420). In the early phases of the Solar System, the orbit of Neptune was slowly expanding due to the injection of planetesimals towards Uranus and Saturn (Fernandez and Ip, Icarus 58, 109). As a consequence, the mean-motion resonances with Neptune slowly migrated to larger semi-major axis, sweeping the inner KB. According to her computations, the vast majority of bodies (including Pluto) should have been adiabatically captured into some mean-motion resonance (mostly the $2 / 3$ and $1 / 2$ ones) and, once captured, their eccentricity should have been adiabatically pumped. This theory explains well the distribution of bodies in the $2 / 3$ resonance with respect to eccentricity, inclination and libration amplitude; nevertheless, it encounters two difficulties. First, it predicts at least as many bodies in the $1 / 2$ resonance as in $2 / 3$ resonance, while none has been presently discovered (see point 3 ). This fact does not seem to be explainable by observational biases. Second, the accretion of bodies of large size such as $1992 \mathrm{QB}_{1}$ indicates (Stern, AJ subm.) that the Kuiper belt should have been much more massive in the past and has been largely depleted. Then, it is likely that the early ages of the inner Kuiper belt were very violent ones, with $99 \%$ of the mass being ejected, so that very gentle phenomena of adiabatic capture into resonances should have been largely dominated by collisional processes, kicking bodies continuously in and out of resonances and stirring up the eccentricities.

Following this idea, an alternative theory to Malhotra's one concerning the origin of Pluto has been advanced by Levison and Stern (Icarus 116,315). They first observe that Pluto is presently in the Kozai resonance inside the $2 / 3$ resonance on an orbit which is stable over the age of the Solar System, but is not far from the edge of the strongly chaotic region, since its amplitudes of libration of the critical angles are quite large. Then, they argue that Pluto was originally on a chaotic orbit at the edge of the $2 / 3$ resonance and was finally kicked into its present stable orbit by a strong collision, possibly originating also the Pluto-Charon binary. 
Concerning point (2), i.e. the absence of bodies on low eccentricity stable orbits between 26 and 40 $\mathrm{AU}$, it is likely that something must have pumped up the eccentricities in the past. This was possibly due to a strong collisional primordial activity or to encounters with big bodies now ejected from the Solar System. Levison (KB workshop) suggested also that the slow loss of mass of the inner Kuiper belt would have forced the $\nu_{8}$ secular resonance to sweep the region up to $40 \mathrm{AU}$, pumping up the eccentricities of all bodies so that only the ones protected from Neptune encounters by mean-motion resonances could survive; this would also explain point (1), in alternative to Malhotra's mechanism. It's worth to point out that also in the outer asteroid belt there are regions at low eccentricity which are stable over the age of the Solar System and which are nevertheless mysteriously depleted.

As one sees, the state of the art is still very approximate, with no coherent scenario emerging yet from the sea of theories and conjectures. What seems to be clear is that a good understanding of the distribution of bodies in the Kuiper belt cannot be achieved simply basing on the present dynamical properties of the trans-Neptunian regions, but should rely also on a consistent model of formation of the Solar System.

\section{B. Dynamics of Planetary Growth}

(G.R.Stewart \& J.J.Lissauer)

Current models of solar system formation hypothesize that the terrestrial planets and the cores of the gas giant planets were formed by the mutual accretion of solid planetesimals. Planetesimals in the inner solar system were made primarily of rocky material (including metals), whereas those farther from the Sun also contained ices. Most planetesimal growth models attempt to reproduce the configuration of planets observed in our solar system, but the ultimate goal is to understand the physical processes involved well enough to model a diverse range of possible planetary configurations (Lissauer, Icarus 114, 217). Important issues for the general problem of planetary growth include the formation of protoplanetary disks, the growth of kilometer-size planetesimals from dust (Weidenschilling and Cuzzi, Protostars \& Planets III, 1031), the accretion of gas by giant planet cores (Pollack et al, Icarus in press) and dispersal of protoplanetary disks.

This report concentrates on the dynamical aspects of the growth of solid bodies from kilometer-sized planetesimals to terrestrial planets and giant planet cores. Bodies in this size range move in perturbed heliocentric orbits, with the principal perturbations resulting from mutual interactions (physical collisions and gravitational scattering) and, in some situations, gas drag. Physical collisions can result in accretion or fragmentation. Major questions include the rate of planetary growth, the mixing of material condensed in differing parts of the disk, the amount of material lost via ejection to interstellar space, the frequency of giant impacts (which are believed to be responsible for the protolunar disk and collisional stripping of Mercury's mantle and partly responsible for planetary rotation, Dones and Tremaine, Icarus 103, 67), the origin of the asteroid belt and the ultimate "stable" configurations of planetary systems. Lissauer and Stewart (Protostars and Planets III, 1061) reviewed the pre-1993 literature on this subject.

Most numerical simulations of the accretion of planets from planetesimals can be divided into two distinct methods. In the first method, planetesimals are modeled as a statistical ensemble that is characterized by a discrete size distribution and velocity distribution. Analytical formulae are used to calculate the collision rates and the velocity evolution of the distribution. As demonstrated by Wetherill and Stewart (Icarus 106, 190), this semi-analytic method is computationally efficient enough to model broad size distributions of planetesimals ranging over 20 orders of magnitude in mass and accretion timescales exceeding $10^{5}$ years. Wetherill and Stewart report that planetary embryos of mass $10^{26}$ grams are formed on a $10^{5}$ year timescale at a distance of $1 \mathrm{AU}$ from the Sun. The rapid growth of planetary embryos is regulated by the collisional fragmentation of smaller planetesimals. The velocities of these collisional fragments are reduced by gas drag, facilitating their capture by the planetary embryos. Omitting fragmentation from the calculation increases the growth time to about $3 \times 10^{5}$ years.

An alternative method of simulating planetary accretion is the n-body simulation. Although n-body simulations lack the efficiency to model broad size distributions of planetesimals, they are well-suited for testing and extending the many approximations that are used in the semi-analytic method. N-body simulations that neglect collisions but include gravitational interactions can be used to study the interactions between planetary embryos and a population of smaller planetesimals. Ida and Makino (Icarus 
$106,210)$ found that a single embryo can dominate the velocities of the smaller planetesimals included in an annulus of radius $a$ and width $d a$ that is about 20 times the Hill sphere radius of the embryo if the mass of the embryo exceeds the quantity $m \sqrt{n \pi a \cdot d a}$, where $m$ is the mass of a small planetesimal and $n$ is the surface number density of small planetesimals in the annulus. When two or more embryos are included in the simulation, Kokubo and Ida (Icarus 114, 247) found that the embryos tend to repel one another until their orbital separation is about 5 mutual Hill sphere radii. This occurs when most of the mass is in the small planetesimals because embryo-embryo interactions increase both eccentricities and semi-major axes separations whereas embryo-planetesimal interactions damp the eccentricities of the embryos without having a large effect on their semi-major axes. This effective repulsion between embryos would tend to delay the merger of embryos into planets until the total number of planetesimals was sufficiently depleted by accretion onto the embryos.

$\mathrm{N}$-body simulations that included physical collisions and accretion were first reported by Aarseth et al (ApJ 403, 351). This work simulated a local box in the solar system by using sheared periodic boundary conditions. Aarseth et al found that runaway growth of the largest planetesimals on a shorter timescale than found by the semi-analytic calculations of Wetherill and Stewart, but most of Aarseth et al's results were based on two-dimensional simulations which tend to shorten collision timescales. Recently, Kokubo and Ida (Icarus 123, 180) describe three-dimensional n-body simulations of planetesimal accretion that decrease the accretion timescale by increasing the planetesimal radii by a factor of five. Kokubo and Ida also find runaway growth of planetary embryos. In order to make a direct comparison with the accretion timescales predicted by the semi-analytic and n-body simulations, Wetherill et al (Lun.Plan.Sc. 27, 1425) have increased the physical radii of planetesimals by a factor of five in the semi-analytical model and have found the time evolution of the velocity and mass distribution of planetesimals to be roughly comparable to the evolution found using Kokubo and Ida's n-body simulation.

Dynamical simulations have been quite successful in reproducing the basic configuration of the inner solar system (Wetherill, Ann. Rev. Earth Planet. Sci. 18, 205) and jovian resonances may be able to account for the asteroid belt (Wetherill, Icarus 100,307). Analogous simulations are less successful in the outer solar system, because the combination of high escape velocities from giant planet surfaces and low heliocentric orbit velocities allow gravitational scattering to produce much more eccentric planetary orbits than are observed (Lissauer et al, Neptune and Triton, 37). However, by adding an artificially enhanced gas drag, which promotes temporary capture into exterior mean-motion resonances with Jupiter, Beaugé et al. (MNRAS 270, 21) were able to simulate the accretion of Saturn's solid core. A more realistic eccentricity damping mechanism for large planetesimals in the outer solar system may have been selfexcited disk torques in the solar nebula (Hahn and Ward, Lun.Plan.Sc. 27, 479; Ward, Icarus 106, 274).

Two explanations have recently been proposed to explain the origin of Pluto's peculiar orbit: If the jovian planets suffered a substantial orbital migration as a result of planetesimal scattering, Neptune could have captured Pluto into the 2:3 orbital resonance and pumped up its eccentricity to its current value (Malhotra, Nature 365, 819). Alternatively, Levison and Stern (Icarus 116, 315) find that gravitational interactions with the current jovian planets can temporarily capture Pluto into the $2: 3$ orbital resonance with Neptune, but a dissipative event, such as the giant impact which presumably formed Charon or gravitational interactions with small bodies in the Kuiper Belt, is required to damp Pluto's libration amplitude and thereby stabilize its resonant orbit.

Close approaches of planetary bodies usually lead to highly chaotic orbital evolution and ultimately to either physical collisions or ejection of material to interstellar space. Thus, in a "mature" planetary system, orbits of the major planets will generally be well-separated, either in distance from the star or as the result of a protective resonance (e.g., Pluto). Chambers et al (Icarus 119, 261) have studied systems of several planetesimals on nearby nonintersecting orbits. They found that the time required for orbits to first cross increases quite rapidly as initial orbital radii are moved apart, but even systems in which all individual planetesimal pairs are significantly farther apart than required for indefinitely stable orbits (cf. Gladman, Icarus 106, 247) are unstable on time scales short compared to the age of the solar system. Duncan and Lissauer (Icarus in press) find analogous results when the masses of the secondaries are varied instead of their orbital spacing. A first step towards a detailed understanding of these results is the successful derivation of a four-dimensional algebraic mapping that closely approximates the chaotic dynamics of the two-planet problem in the limit of small orbital separations (Stewart, Physica D in 
press). Long-range perturbations, chaos and orbital stability criteria are thus likely to be major factors in determining the "ultimate" configurations of planetary systems. "Stability" in this sense means that no drastic changes are likely to occur for the time scale of interest, e.g., the star's main sequence lifetime; even our "mature" planetary system is almost certainly unstable on extremely long time scales (Laskar, AA 287, L9).

\section{Relativity, Cosmology and Celestial Mechanics}

(G.Contopoulos)

In recent years there has been much interest on problems of chaos in General Relativity and Cosmology. These problems require the use of methods developed in Dynamical Astronomy and constitute an interface of interaction of Dynamical Astronomy with Relativity and Cosmology.

An earlier review of this subject was written by Barrow (Phys. Rep. 85, 1, 1982). A more recent review can be found in "Deterministic Chaos in General Relativity" (NATO Adv. Stud. Inst. Ser. B Phys. 332, 1994). Besides a mathematical section, this book deals with specific cases where chaos is important. Such is the case of two fixed black holes (Contopoulos). This problem is integrable in the Newtonian limit (two fixed centers), but it is completely chaotic in the case of photons in General Relativity (chaotic scattering), while the case of particles of non-zero rest mass contains both ordered and chaotic orbits. Chaos appears also in perturbed black holes (Bombelli) and in relatively simple cosmological models (Calzetta, Ellis, Tavakol and Tomaschitz; see also Calzetta and El Hasi, Class. Quantum Grav. 10, 1825; Phys. Rev. D. 51, 2713). A study of special interest refers to the use of fractals in Cosmology (Ribeiro). A large section of this book is devoted to the dynamics of the Mixmaster Model of the Universe, introduced by Misner (1969) and Belinskii, Khalatnikov and Lifshitz (1969-1970). Several articles are devoted to the possibility of chaos in this model. This problem is puzzling because: (a) it looks very simple mathematically (it can be written as a system of six first-order differential equations with quadratic second members); (b) there are some indications of chaos, yet the maximal Lyapunov characteristic number is zero (Hobill et al). Contopoulos, Grammaticos and Ramani have found a general solution depending on 6 arbitrary constants satisfying the Painlevé property (i.e. its singularities in complex time are only poles). Furthermore, Cushman and Sniatycki (Rep. Math. Phys. 36, 75) have shown that the Mixmaster model has no recurrences, thus it is not chaotic in the usual sense.

This problem has been solved by Latif, Musette and Conte (Phys. Lett. 194A, 83) and by Contopoulos, Grammaticos and Ramani (J. Phys. A28, 5313), who found that there are special solutions of the Mixmaster model that do not have the Painlevé property. This indicates that this model is not integrable. As regards the Lyapunov characteristic number, it is zero because all orbits escape to infinity. But the finite time Lyapunov characteristic number is always positive. The conclusion is that the Mixmaster model is chaotic in the sense of chaotic scattering.

The renewed interest in chaotic models of the Universe is due to the fact that such models may provide a mixing of the early universe that solves the "causality problem" without needing inflation. As the problem of inflation and the amount of dark matter is still unsettled, such alternatives are important. Several alternatives to dark matter have been proposed in recent years that invoke a change of Newton's law of gravitation. A far-reaching hypothesis was introduced by Kazanas and Mannheim (ApJ 342, 635; Phys. Rev. D44, 417; ApJ Supp. 76, 431; Gen. Rel. Grav. 26, 337) who replaced Einstein's field equations by using Weyl's tensor instead of the Ricci tensor. This "conformal gravity" theory leads to field equations of motion that are of $4^{\text {th }}$ order, instead of the usual $2^{\text {nd }}$ order (but the equations of motion are of $2^{\text {nd }}$ order). Despite this complication, this theory has many attractive properties, e.g., it gives an asymptotically flat rotation curve for a point mass, without requiring any cosmological dark matter. Further discussions can be found in "Nonlinear Problems in Relativity and Cosmology" (Ann. N.Y. Acad. Sc. 631, 1991).

In recent years, much interest in nonlinear dynamical systems was shown by elementary particle physicists that developed theories to unify the various forces of nature in the very early universe. E.g., the analogy of solitons with monopoles (products of the Grand Unified Theories) and elementary particles in general was stressed by several authors. Nonlinear phenomena, like intermittence, play an important role. Finally, we should mention the efforts of Penrose (e.g., in Hawking and Israel, eds., "Three hundred years of Gravitation", Cambridge, 1987, p.17) to develop a theory that should unify gravity with the 
other forces of nature, not by quantizing gravity, as most theories try to do, but by introducing gravity in a nonlinear version of quantum mechanics. This approach requires the full use of the methods of nonlinear classical mechanics into quantum mechanics.

An important book that compares the classical and quantum approach to chaos is Gutzwiller's "Chaos in Classical and Quantum Mechanics" (Springer Verlag, New York, 1990). Further new developments in Relativity and Cosmology that are related to chaos can be found in physics journals like: Journal of Mathematical Physics (Amer. Inst. Physics), Classical and Quantum Gravity (Inst. of Phys. Bristol, U.K.), Journal of Physics A. Mathematical and General (Inst. of Phys., Bristol, U.K.) and Physical Review D. Particles, Fields, Gravitation and Cosmology (Amer. Inst. Physics).

\section{Determination of Relativity Parameters \\ (X X Newhall)}

The past three years have witnessed a number of experiments yielding refined values of relativity parameters, all of which seem in concert with the predictions of General Relativity (GR). The most significant source of the new results was Lunar Laser Ranging (LLR) data, for three reasons: There are now three more years of accumulated data; the accuracy of recent data has been increasing, with observational errors $\lesssim 2-3 \mathrm{~cm}$; the increased span allows better separation of relativity parameters from others with which they are more correlated over a shorter span. Other results arose from the analysis of spacecraft signals, Mercury radar ranging, and very-long-baseline interferometry (VLBI) data.

\section{THE PRINCIPLE OF EQUIVALENCE}

Nordtvedt has pointed out that a violation of the Principle of Equivalence would cause a polarization of the lunar orbit along the Earth-Sun direction. In a pair of papers, Williams, Newhall and Dickey [WND] (Phys. Rev. D 53, 6730; Science 265, 482) analyze the sensitivity of LLR data to a number of relativity parameters, among them Nordtvedt's dimensionless parameter $\eta$, a scaled measurement of the departure from unity of the ratio of gravitational mass to inertial mass of any system. When solar radiation pressure is accounted for, and assuming no violation of the Weak Equivalence Principle, the result for the difference of the Earth's and Moon's gravitational-to-inertial mass ratios gives $\eta=-0.0007 \pm 0.0010$. Chandler, Reasenberg and Shapiro (Bull. AAS 26, 1019) derive an identical uncertainty in $\eta$ using LLR, spacecraft tracking data, planetary radar, and VLBI observations. Orellana and Vucetich (AA 273, 313) use observations of the Trojan asteroids to test the equivalence principle. The Trojans act as resonators for long-period perturbations and would be sensitive to any violation of the equivalence principle. They estimate $\eta=-0.56 \pm 0.48$.

\section{THE PPN SUPERPOSITION PARAMETER $\beta$}

The papers WND derive two estimates for $\beta$ from LLR data. The first is directly from the appearance of $\beta$ in the equations of motion. When estimated concurrently with $\gamma$, both uncertainties are 0.005 , though a correlation of -0.86 shows that the combination $\beta+\gamma$ is better determined, with an uncertainty of 0.003 . The second estimate uses Nordtvedt's relation $\beta=(\eta+\gamma+3) / 4$. In an earlier paper, Reasenberg et al (ApJ 234, L219) use interplanetary ranging to establish that $\gamma=1.000 \pm 0.002$. Incorporating this result and the above uncertainty for $\eta$, the estimate is $\beta=0.9999 \pm 0.0006$.

\section{THE PPN CURVATURE PARAMETER $\gamma$}

Several papers give results involving $\gamma$. WND again use LLR data to find that $\gamma=1.000 \pm 0.005$, an estimate done simultaneously with $\beta$. Lebach et al (Phys. Rev. Lett. 75, 1439) use VLBI observations of extragalactic radio sources to measure the gravitational deflection of radio waves by the Sun. Their result is $\gamma=0.9996 \pm 0.0017$, corresponding to a gravitational bending of $0.9998 \pm 0.0008$ times that predicted by GR. In another paper, Damour and Vokrouhlický (Phys. Rev. D 53, 4177) show by power series representation of the lunar orbit that a violation of the Equivalence Principle amplifies the leading term by $62 \%$ and that there are orbits for which the amplification becomes unstable. They present the result from a string-derived non-Einsteinian theory that analysis of LLR data yield $\gamma-1=(-0.9 \pm 1.3) \times 10^{-7}$, 
though it is cautioned that this small uncertainty cannot be compared directly with those above. Pitjeva (CMDA 55, 313), uses radar ranging to Mercury to estimate a topographical model and other parameters, including a result for the combination $\nu=(2+2 \beta-\gamma) / 3=0.9995 \pm 0.0013$. If $\beta$ is assumed to be unity, she finds that $\gamma=1.0002 \pm 0.00096$.

\section{VARIATION OF THE GRAVITATIONAL "CONSTANT" $\dot{G} / G$}

The WND papers derive the result $\dot{G} / G=(1 \pm 8) \times 10^{-12} \mathrm{yr}^{-1}$. The analysis presented shows that the effect of $\dot{G} / G$ on the lunar orbit is highly correlated with that of Earth tides over short time scales, but that quadratic terms cause the effects to separate over the longer data spans now available. The main reason is that solar perturbations of the lunar orbit have signatures distinct from those of the tides. Pitjeva (op cit) uses the Mercury radar observations to determine $\dot{G} / G=(4.7 \pm 4.7) \times 10^{-12} \mathrm{yr}^{-1}$.

\section{GEODETIC PRECESSION}

For the lunar orbit, the geodetic precession predicted by GR appears as a precession of the node and of the longitude of perigee along the ecliptic, which in turn is dominated by the inclination of the lunar orbit to the ecliptic. The recent full set of LLR data allows determination of the orientation of these planes very well. In the WND paper, those terms in the equations of motion which cause the node to precess were isolated and a possible departure from GR was estimated. The result found was that the observed geodetic precession differs from the GR value by a factor of $-0.003 \pm 0.007$. The highest correlation is +0.56 with the lunar $J_{2}$.

\section{THE SOLAR REDSHIFT}

Another test of the Equivalence Principle was done using the signal from the Galileo spacecraft. Krisher, Moribato and Anderson (Phys. Rev. Lett. 70, 2213) analyzed the signals received from the ultra-stable oscillator for about 600 days following launch. They estimated the effects both of the complete relativistic portion of the expression for received frequency and of the portion due only to gravitational potential. The total frequency shift was consistent with GR to $0.5 \%$; the contribution from gravitational potential (the solar redshift) was consistent to $1 \%$.

\section{ACCELERATION OF THE EARTH AND MOON BY DARK MATTER}

It has been proposed that dark matter constitutes perhaps as much as $90 \%$ of the galactic mass. As another test of a possible violation of the Equivalence Principle, Nordtvedt, Müller and Soffel (AA 293, L73) use LLR data to test for differential acceleration of the Earth and Moon arising from compositiondependent coupling with the dark matter. Any such effect will appear as a polarization of the lunar orbit in the direction of the acceleration. Their result is that the amplitude of this polarization is $\leq 1.1 \mathrm{~cm}$, implying a limit of $3 \times 10^{-14} \mathrm{~cm} / \mathrm{sec}^{2}$ for any differential acceleration.

\section{E. The Motions of the Moon}

(S.Ferraz-Mello)

The growing set of accurate lunar distance measurements over the past few years has stimulated an increased interest in the study of the motions of the Moon, both orbital and rotational. The full utilization of these data to understand the physics underlying these motions can be done only if highprecision theories are available to provide exact reflector coordinates.

The new set DE403/LE403 of integrated planetary and lunar ephemerides established at JPL (Standish et al, JPL IOM 314.10-127; Standish and Newhall, IAU Symp. 172, 29; Newhall et al, IAU Symp. $172,37)$, spanning 6000 years, is based on the relativistic n-body equations of motion for the Sun, Moon and planets, the 3 major asteroids perturbing the Moon and planets, and the resultant accelerations of the Earth, Moon and Mars due to 297 additional asteroids. The comparison of these ephemerides with observations allowed Newhall et al (see the previous report) to determine the main physical parameters related to the foundations of the General Theory of Relativity. The analytical theory ELP2000-82B with 
a numerical complement has been adjusted to fit the JPL ephemerides at the centimeter level and LLR observations (Chapront and Chapront-Touzé, IAU Coll. 165).

Regarding the rotation of the Moon, the progress was due mainly to numerical simulations of improved models. Both orbital theories mentioned above were accompanied by integration of the three Euler angles describing the lunar physical librations. Chapront and Chapront-Touzé (op cit) improved the lunar libration theory of M. Moons to be consistent with ELP and adopted a new tidal model. The DE403/LE403 ephemerides also include the Moon's librations and were fit to a 25-year span of lunar laser ranging measurements. The model used for the librations in the integration includes the interaction of the lunar figure (including solid tides) with the figure of the Earth and with the point-mass Sun, Venus, Earth and Jupiter. Also modeled is tidal dissipation in the lunar mantle (Newhall et al, op cit).

Comparison with observations revealed a large rotational dissipation of uncertain origin, consistent with either tidal effects or viscous damping at the surface of a small fluid core interacting with the mantle (Dickey et al, Science 265, 482). It also confirmed the existence of a 2.9-yr oscillation of the zero lunar longitude about the mean direction to the Earth, with amplitude in the range of zero to 1.5" and a 75-yr wobble of the pole direction, analogous to the Earth's Chandler wobble (Williams et al, Lun.Planet.Sc. 27, 1439).

A selected section of the numerical integration, spanning 718 years, was Fourier analyzed. The free libration amplitude is 1.8" (but blended with two forced amplitudes due to Venus). Such an amplitude could have been stimulated by impacts, internal processes or the passage through a resonance. As the mean longitude of the Moon accelerates, planetary perturbation terms may become resonant. There is one term in ELP2000 whose amplitude is only 0.21 mas, but it passed through a resonance 33 to 66 thousand years ago and could be the source of the free libration term (Eckhardt, CMDA 57, 307).

A continued series of investigation on the rotation of the Moon in agreement with the requirements of current observational accuracy was conducted by Bois et al (Wytrzysczak and Bois, NATO Adv. Stud. Inst. Ser. B Phys. 272, 257 and 265; Bois et al , CMDA 53, 185; Bois and Journet, CMDA 57, 295; Bois and Vokrouhlicky, AA 300, 559; Journet and Bois, CMDA in press). They have integrated the relativistic $n$-body equations of motion for the Sun, Moon, planets and the 5 major asteroids perturbing the planets, and taking into account the figures of the Earth and Moon, their tides, internal structures, elastic and anelastic deformations. These simulations included all direct perturbations capable of producing perturbations in the Moon's rotation with amplitude larger than 0.1 mas. Relativistic librations with amplitudes as large as 1 mas, not negligible with respect to the current accuracy of the observations, were found. The spin-orbit coupling and the presence of a core inside the Moon related to the mantle by inertial coupling proved to be adequate to excite the two resonant frequencies of the lunar physical librations, namely 2.9 and 80.1 years, without having recourse to external causes. The discussion follows a new general classification of the librations (Bois, AA 296, 850).

A 600-yr integration of the relativistic equations of the rotation motions of the Earth and the Moon was also done by Eroshkin and Pahkevich (IAU Coll. 165), and precise short-term integrations for the sake of comparing to observations by Aleshkina et al (IAU Coll. 165).

The long-term tidal evolution of the Earth-Moon system was reexamined by Touma and Wisdom (AJ 108, 1943). Classical results on the history of the lunar orbit were confirmed by their more general model. The increase of the mean motion of the Moon due to long-period ocean tides was determined by Cheng et al (Geoph. Res. Int., 108, 401). The secular variation of the Moon's rotation rate was investigated by Bois et al (AA in press).

The author thanks E. Bois, J. Chapront and X X Newhall for their comments on the first version of this report.

\section{F. The Impact of Modern Mathematics in Celestial Mechanics}

\section{(A. Giorgilli)}

According to Poincaré, the general problem of dynamics is the study of small perturbations of an integrable Hamiltonian system (Méthodes Nouvelles de la Mécanique Céleste, chap. I, N. 13). The particularly interesting case is an Hamiltonian of the form $H(p, q ; \varepsilon)=h(p)+\varepsilon f(p, q)$, where $p, q$ are action-angle variables and $\varepsilon$ is a small parameter. For $\varepsilon=0$, the phase space is foliated into invariant $n$-dimensional 
tori (where $n$ is the number of degrees of freedom) carrying periodic or quasi periodic motions, depending on the existence of resonance relations among the frequencies $\omega(p)=\partial h / \partial p$.

In very rough terms, the typical attempt is to prove that the perturbed system (i.e., the case $\varepsilon \neq 0$ ) is still integrable in the sense of Liouville. Most attempts in the past century were performed with the aim of computing actual orbits as perturbations of the orbits of the unperturbed system. After the work of Poincare and Birkhoff, a standard tool is the reduction of the system to a normal form. These methods turn out to be very useful as far as short-term predictions or some qualitative aspects of the dynamics are concerned. However, Poincaré proved that a perturbed system is generically non integrable in Liouville's sense (Méth.Nouv., chap. V). Concerning the classical methods of solution by series, Poincaré proved that divergence is the general rule, with only one possible exception: the series of Lindstedt in case the frequencies obey some suitable nonresonance condition. To be precise, he failed to prove divergence in this case, but considered convergence as "fort invraisemblable" (Méth.Nouv., chap. XIII, N. 149). Finally, he discovered the phenomenon of homoclinic or heteroclinic intersections between the stable and unstable manifolds of periodic orbits, which lies at the basis of the chaotic behaviour (Méth.Nouv., chap. XXXIII).

After Poincaré (and after the discovery of Quantum Mechanics), the study of the "general problem of dynamics" has been essentially confined to the field of pure mathematics for half a century. There are exceptions of course: the most remarkable is perhaps the book of M. Born "The Mechanics of the Atom", Frederick Ungar Publ. Co., 1927. In crude terms, the interest of physicists and astronomers for dynamical systems has been awaked by the announcement of the theorem of Kolmogorov and (perhaps mainly) by the introduction of modern computers.

It is worth mentioning a few historical points (by the way, this reflects the experience of the author of this report, without pretending to be exhaustive). The first numerical experiment by Fermi, Pasta and Ulam was performed in 1954 (Los Alamos doc. LA-1940). In the same year, Kolmogorov announced his theorem, on the persistence of invariant tori, in a short memoir including a sketch of the proof (Dokl. Akad. Nauk SSSR, 98, 527). At the beginning of the sixties, Contopoulos made an extensive study of the applicability of formal integrals to galactic models (Z.Ap 49, 273; AJ 68, 1 and AJ 70, 526). In the same years, Moser and Arnold produced a complete proof of Kolmogorov's theorem (J.K. Moser, Nachr. Akad. Wiss. Gött., II. Math.-Phys. Kl. 1962, Nr.1; V.I. Arnold, Russ. Math. Surv. 18, 9, 1963). Moreover, the first and, for a long time, sole example of instability in Hamiltonian systems, thereafter called "Arnold diffusion", was discovered by V.I. Arnold (Sov. Math. Dokl. 5, 581). The existence of chaotic motions even in simple system became evident to everybody after the work of Hénon and Heiles (AJ 69, 73, 1964). The mechanism of overlapping of resonances was identified by Contopoulos (Bull. Astr. Ser. 3, 2 , $223,1967)$.

Starting from the beginning of the seventies, the interaction between mathematics and applied sciences, including in particular physics and astronomy, became deeper and deeper: the exponential growth of interest - and of the number of publications - makes it impossible to continue the list above. On the other hand, a complete discussion of this subject is far beyond the limits of this report, of course. A rather complete collection of recent mathematical results can be found in the proceedings of the NATO Adv. Stud. Inst. "Hamiltonian systems with three or more degrees of freedom" (C. Simó ed., Kluwer, Dordrecht, in press), hereafter referred to as 3DHAM95.

Here, attention will be concentrated on three topics: (1) quasiperiodic solutions on invariant tori, (2) exponential stability, and (3) splitting of separatrices and Arnold diffusion.

\section{QUASIPERIODIC SOLUTIONS ON INVARIANT TORI}

The KAM theory has been considerably developed after the original memoir of Kolmogorov (e.g.: regularity conditions for the existence of KAM tori; Aubry-Mather theory; optimality of the nonresonance conditions; infinite systems; non Hamiltonian systems; etc.). A recent survey concerning the existence of invariant tori can be found, for instance, in the contribution of H.W. Broer, G.B. Huitema and M.B.Sevryuk to the proceedings of the conference "Nonlinear dynamical systems and chaos" (H.W. Broer et al, eds., Birkhäuser, 1996). Attention will be paid here to recent works, close enough (in some, perhaps, questionable sense) to Celestial Mechanics. For numerical works inspired by KAM theory, see the contributions to previous IAU Commission 7 reports of J. Laskar (Trans. IAU 21A, 16) and of A. Milani and S. Ferraz-Mello (Trans. IAU 22A, 20). 
The main interest of KAM theory in Celestial Mechanics is connected with the problem of perpetual stability of orbits in the solar system. We recall in a few words the statement of KAM theorem. If the size $\varepsilon$ of the perturbation is small enough, then the unperturbed tori characterized by strongly nonresonant frequencies survive under perturbation and fill up a set of large relative measure in the phase space. However, the complement of the invariant tori is open and dense and, for more than 2 degrees of freedom, it is connected, too. Thus, stability can be assured only in probabilistic sense.

Quasiperiodic solutions lying on invariant tori can be constructed with the method of LindstedtPoincaré (Méth.Nouv., chap. IX). By KAM theorem, these series are actually convergent in case the frequencies satisfy a suitable irrationality condition. However, they are not absolutely convergent (J. Moser, Math. Ann. 169, 136). The problem is that the algorithm of Lindstedt-Poincare produces terms which grow too fast to allow for absolute convergence, due to a bad accumulation of small divisors. Thus, convergence can not be established via traditional methods, for instance, the method of majorants due to Cauchy. However, there are cancellations among such big terms, which account for the convergence of the series (H. Eliasson, MPEJ - Math. Phys. Electronic Journal, 2, Nr.4). The structure of the series of Poincaré-Lindstedt is better investigated using a tree representation for the coefficients generated by the algorithm. Collecting the so called "resonant trees" (corresponding to terms with a bad accumulation of small divisors) allows one to produce an explicit resummation algorithm for the series, from which convergence follows (G. Gallavotti, Comm. Math. Phys. 164, 145; L. Chierchia and C. Falcolini, Ann. Sc. Norm. Sup. Pisa Cl. Sci. 21, 541). For a recent report on this matter, see G. Gallavotti (3DHAM95, in press) and L. Chierchia and C. Falcolini (3DHAM95, in press).

An alternative approach consists in implementing the construction of Kolmogorov's normal form for the Hamiltonian using Lie series expansions. An accurate analysis shows that there is no bad accumulation of small divisors, so that the algorithm produces absolutely convergent series (A. Giorgilli and U. Locatelli, 3DHAM95, in press). However, going through the construction of a normal form makes the algorithm less efficient from a practical viewpoint.

For simple systems, for instance the standard map and the forced pendulum, the construction of the series of Lindstedt can be explicitly performed via algebraic manipulation on computers. The series so constructed can be used in computer assisted proofs. In some cases these methods give rather good estimates for existence of tori. A survey on this subject has been published by A. Celletti and L. Chierchia (Dyn. Rep., new ser. 4, 60).

The applications of the KAM theory to models of interest in Celestial Mechanics is still limited to a few examples. The existence of librational invariant surfaces plays a relevant role in understanding the spin-orbit problem (A. Celletti, NATO Adv. Stud. Inst. Ser. B Phys. 331, 229). The problem of stability for the solar system (or at least for some relevant part of it) is still far from being solved via the KAM theory. Even in the simple case of the Sun-Jupiter-Saturn system the estimated threshold for existence of invariant tori is still too small (P. Robutel, Thèse, Univ. Paris; J. Laskar and P. Robutel, CMDA 62, 193 and 266).

Numerical computations over a very large time interval show that the motion of the major planets is very close to quasiperiodic. On the other hand, numerical computation show also that the motion of the internal planets is far from quasiperiodic (see, for instance, J. Laskar, Icarus 88, 266; see also Laskar's contribution to the 1990 IAU Commission 7 report, Trans. IAU 21A, 16). Thus, it appears that the dynamical behaviour of the solar system can not be fully understood in the framework of KAM theory. In particular, perpetual stability in the sense of KAM theory is excluded, if not in rigorous mathematical sense, at least in view of numerical results. The KAM approach seems instead to be applicable to a reduced model including only the Sun and the major planets.

\section{EXPONENTIAL STABILITY}

For practical application, including the problem of stability of the solar system, perpetual stability is not mandatory. Proving that the inclinations, eccentricities and semi-major axes of the planets can not significantly change for a time interval comparable with the estimated age of the solar system would be enough, of course. Remark that the latter quantities are essentially the action variables of the Keplerian problem. The theory of adiabatic invariants deals exactly with this problem: the typical result is that the actions of the system do not change significantly for a time $O(1 / \varepsilon)$. 
In the Hamiltonian case, we can do better: for $\varepsilon \rightarrow 0$ the stability time increases faster than any inverse power of $\varepsilon$. This has been first pointed out by J.K. Moser (Nachr. Akad. Wiss. Gött., II. Math.Phys. Kl. 1955, Nr.6) and J. Littlewood (Proc. London Math. Soc. 9, 343, 1959). The name "exponential stability" has been originated by the work of N.N. Nekhoroshev (Russ. Math. Surveys 32, 1, 1977) and Trudy Sem. Petrovs. 5, 5, 1979). The typical statement is that the action variables change at most by $O\left(\varepsilon^{b}\right)$ for a time $O\left(\exp \left(1 / \varepsilon^{a}\right)\right)$, where $a<1$ and $b<1$ are constants. The exponential dependence of the time on the inverse of the perturbation parameter $\varepsilon$ is the interesting aspect: in the words of Littlewood, "if not eternity, it is a considerable slice of it". A self contained, shorter proof Nekhoroshev's theorem has been given by G. Benettin, L. Galgani and A. Giorgilli (CMDA 37, 1). An interesting approach based on the theory of simultaneous approximations going back to Dirichlet has been proposed by P. Lochak (Usp. Math. Nauk. 47, 59 or Russ. Math. Surv. 47, 57; Nonlinearity 6, 885).

The result follows from a careful analysis of the structure of resonances in action space. One considers only resonances of finite order. A resonance originates a resonant manifold and deeply influences the dynamics on a small region around that manifold causing a fast motion (with velocity $O\left(\varepsilon^{b}\right)$ ) along a particular plane, named by Nekhoroshev the "plane of fast drift". With some hypothesis on the unperturbed Hamiltonian (convexity or steepness) one assures that the plane of fast drift is transversal to the resonant manifold, so that the motion tends to push the orbit out of the resonance. If resonances of the same multiplicity do not overlap, then one can prove that the motion is actually confined inside the resonance for an exponentially long time. The dynamics inside resonant regions can be very complicated. In particular, the existence of regions of chaotic motions must be considered as typical (G. Benettin and G. Gallavotti, J. Stat. Phys. 44, 293). Actually, the size of the region associated to a resonance decreases exponentially with the order of the resonance, so that the fundamental property of non overlapping of resonances can be satisfied. Increasing the perturbation parameter causes resonances to overlap and create paths of fast diffusion from one resonance to the next one (G. Contopoulos, Bull. Astr. Ser. 3, 2, 223; B.V, Chirikov, Phys. Rep. 52, 263).

Exponential stability estimates turn out to be particularly simple if one considers the neighbourhood of an elliptic equilibrium, where the unperturbed system can be reduced to a system of harmonic oscillators (A. Giorgilli, Ann. Inst. H. Poincaré Phys. Théor. 48, 423; A. Giorgilli, A. Delshams, E. Fontich, L. Galgani and C. Simó, J. Diff. Eqs. 77, 167; A. Delshams and P. Gutiérrez, 3DHAM95, in press). A similar argument applies to the neighbourhood of a KAM torus: in this respect, an elliptic equilibrium with diophantine linear frequencies and a KAM torus behave the same way. The perturbation parameter in both these cases is the distance from the equilibrium, or from the invariant KAM torus. One proves a local property of exponential stability. Precisely, an orbit starting at a distance $\rho$ does not escape from a neighbourhood of size, say, $2 \rho$ for a time as long as $\exp \left(1 / \rho^{1 / n}\right)$.

As a matter of fact, the neighbourhood of a KAM torus (or of an elliptic equilibrium) turns out to be superexponentially stable. Indeed, a repeated application of KAM and Nekhoroshev's theory leads to the conclusion that it contains a subset of invariant tori of measure $O\left(1-\exp \left(1 / \rho^{1 / n}\right)\right)$ and that the escape time can be estimated from below as $O\left(\exp \left(\exp \left(1 / \rho^{1 / n}\right)\right)\right)$ (A. Morbidelli and A. Giorgilli, J. Stat. Phys. 78, 1607). This result is still local. Concerning the global aspect, in the whole phase space there is a hierarchic structure of nested domains underlying the phenomenon of diffusion. At each level the diffusion speed is exponentially small with respect to the previous level. The set of KAM tori is the domain characterized by a zero diffusion speed (A. Morbidelli and A. Giorgilli, Physica D 86, 514; A. Giorgilli and A. Morbidelli, ZAMP, in press; see also A. Delshams and P. Gutiérrez, J. Diff. Eq., 128, 415).

Applications of Nekhoroshev's theory to specific models are still quite rare. Analytical theories have ben developed for the fast rotations of a rigid body (F. Fassò, CMDA 62, 43; G. Benettin and F. Fassò, Nonlinearity 9,$137 ; \mathrm{F}$. Fassò, ZAMP, in press). The problem of trapping of particles in a strong, non uniform magnetic field has been investigated by G. Benettin and P. Sempio (Nonlinearity 7, 281). The Keplerian case has been investigated by L. Niedermann (Thèse, Université de Paris). The dynamics in the asteroid's main belt has been investigated by A.Morbidelli and M. Guzzo (CMDA subm.). It turns out that uniform estimates are impossible, but one can isolate regions characterized by resonances of sufficiently high order where Nekhoroshev's type results can be proven. This analysis could account for many aspects in the distribution of the asteroids.

Computer assisted analysis has been developed mainly for the restricted problem of three bodies. 
However, as in the case of KAM theory, the results are still typically far from being realistic. In the case of the system Sun-Jupiter-Ceres stability over the age of the solar system is assured only for a mass of Jupiter smaller than the real one by a factor $10^{3}$ (A. Celletti and L. Ferrara, CMDA in press). The best available result concerns the stability of the Lagrangian equilibria: in the case of $L_{4}$ and considering only the planar circular case, stability over the age of the universe is assured in a domain that contains 4 known asteroids out of 98 (A. Giorgilli and Ch. Skokos, AA in press).

\section{SPLITTING OF SEPARATRICES AND ARNOLD DIFFUSION}

As pointed out by Poincaré, chaotic behaviour is generated by homoclinic or heteroclinic intersections between stable and unstable manifolds associated to periodic orbits. The mechanism of heteroclinic intersections has been used by Arnold in his example of a topologically unstable system. The problem of proving that splitting of separatrices and Arnold diffusion are generic phenomena is still open.

The problem is to measure the angle of splitting, which also measures the size of the corresponding chaotic region. The difficulty is that the angle of intersection is exponentially small (A.I. Neishtadt, J. Appl. Math. Mech. 45, 766).

The technology of Poincaré-Melnikov's integrals has been recently adapted to this problem, at least in simple cases. Exponentially small splitting of separatrices has been found in periodically forced systems (A. Delshams and T.M. Seara, NATO Adv. Stud. Inst. Ser. B Phys. 331, 181; D. Sauzin, Ann. Inst. Fourier (Grenoble) 45, 453) and in maps (E. Tabacman, Phys. D 85, 548; A. Delshams and R. RamírezRos, Nonlinearity $\mathbf{9}, 1$ ).

The size of the splitting of separatrices is related to the size of the coefficient of a resonant term in the local Birkhoff normal form of a perturbed system. According to standard estimates of Nekhoroshev type, the size of such a coefficient is generically exponentially small, which accounts for the corresponding exponential smallness of separatrices. This is confirmed by numerical computations (A. Morbidelli and A. Giorgilli, Physica D, in press).

The problem of Arnold diffusion is definitely more difficult. Roughly speaking, one wants to prove the existence of orbits such that the action variables change by $O(1)$ for some (large) $t$. Arnold's idea was to construct a chain of unstable 1-dimensional tori in a system with 2 degrees of freedom and to add a time dependence via a forcing term. The invariant tori have stable and unstable manifolds (named "whiskers" by Arnold) and one proves that there are heteroclinic intersections between the whiskers of nearby tori. This creates a path for diffusion. This example is in some sense artificial, in the sense that it needs two parameters: the first parameter creates the whiskers of the invariant tori; the second one causes the whiskers to form heteroclinic intersections. The second parameter must be exponentially small with respect to the first one.

Proving that this mechanism is typical in Hamiltonian systems is a challenge. The most complete reference is the encyclopædic work of L. Chierchia and G. Gallavotti (Ann. Inst. H. Poincaré, Phys. Théor. 60(1)), where the authors treat the so-called "a priori unstable system". This means that the system contains already two parameters which act like in Arnold's example. The typical case, where there is only one perturbation parameter, is still an open problem (see, however, L. Chierchia, preprint deposited in mp_arch).

An alternative approach has been recently proposed by U. Bessi (Nonlin. Anal., 26, 1115). His suggestion is to prove the existence of diffusion orbits using variational methods. This gets rid of complicated calculations involving Melnikov integrals.

The author thanks G. Benettin, A. Celletti, L. Chierchia, A. Delshams and A. Morbidelli for their suggestions.

Sylvio Ferraz-Mello

President of the Commission 